\title{
ANIMAL STRESS MODELS IN THE STUDY OF STRESS AND STRESS RELATED PHYSIOLOGICAL AND PSYCHOLOGICAL DERANGEMENTS
}

\author{
Urban John Arnold D’Souza, Md Shamsur Rahaman \\ Faculty of Medicine and Health Sciences, Universiti Malaysia Sabah, 88400, Kota Kinabalu, Sabah, Malaysia. \\ *Corresponding Author Email: dsouza@ums.edu.my
}

This is an open access article distributed under the Creative Commons Attribution License, which permits unrestricted use, distribution, and reproduction in any medium, provided the original work is properly cited.

\begin{abstract}
ARTICLE DETAILS
Article History:

Received 12 November 2017 Accepted 12 December 2017 Available online 1 January 2018

ABSTRACT

Animal models of stress were successfully adopted in the stress research over the years. Generally, rodent model is successfully used and since, they can be utilized for studying different stress stimuli such as psychological, somatic or physical and a combination of both. Since human beings are difficult to be used to such specified and varied stress stimuli, the results obtained can be extrapolated to few stress situations that may lead into anxiety, depression and psychiatric conditions. The different models used vary from isolation, noise, predator exposure and diurnal variations mainly as psychological models and immobilization, foot shock, temperature variation and unpredictable stress exposure as physical stress models. Between the lines, few psychological models and somatic models may also be a mixture of both as physical discomfort or pain will also have psychological component. Nevertheless, these models have provided a strong basis in understanding the basis of psychological and psychiatric problems that are faced by human beings and contributed in the development of drug development and their testing for the good of mankind.
\end{abstract}

\section{KEYWORDS}

Stress, Psychological, Somatic, Isolation, Restraint, Rodent.

\section{INTRODUCTION}

Stress, tension and anxiety are common in day to day life of every individual. Little stress is always perceived as a key to success whereas stress experience is prolonged it may be distress and further may lead into a variety of physiological and psychological sequelea that may affect the health adversely. Stress experience depends on its severity, duration and perception of the individual. Prolonged stress may result in several psychiatric disorders such as anxiety, depression, mood problems and schizophrenia [1]. Stress activates the hypothalamo-pituitary- adrenal (HPA) axis and that results in over secretion of stress hormones especially the glucocorticoids which is a relevant hormone indicating the stress response [2].

Stress may be physiological, psychological or a combination of both. Stress experience, its perception and outcome may vary from individual to individual. For few stress experiences may not have adverse effects compared with the others with a similar stress stimulus. Stress experience and its perception vary and in a way some kind of resistance and vulnerability matters. Stress perception may be individualized in terms of its adverse outcome. It is very difficult to measure stress outcome among the human beings as extent of stress stimuli, its perception and adverse outcome vary. Medical research is highly dependent on pre-clinical experiments and experimental animal models are the preferred choice and in stress research as well experimental animal models were the preferred choice as individualized stress experience can be gauged, its duration, severity and similarity among groups can be studied scientifically. McEwen et al. demonstrated animal models of anxiety disorders subjected to chronic stress which exhibited behavioral changes [3]. Physiological or somatic and psychological outcomes on exposure to stress and neurobiological, neuro-endocrine, molecular and genetic background involved in stress research gained momentum because of the use of experimental animal models in the stress research. A variety of animal stress models with physical and psychological stress experience with acute and chronic duration exposure were successfully developed and were useful models in extrapolation with clinical data. These models provided a background basis in stress research. Few examples of

psychological stress are; isolation stress, predator exposure stress, noise stress, circadian variations where dark and light (day and night) reversal is done. Physical stress involves, immobilization or restraint, variation in temperature such as hot and cold, electric foot shock and overcrowding etc.

\section{PSYCHOLOGICAL STRESS MODELS}

\subsection{Isolation stress}

Experimental animals, especially rodents such as rats and mice will be isolated in special isolation cages; a cage covered in all the four sides with black paper with only the roof exposed in polypropylene cages [4]. Rats/mice will be left in the cages individually for a specified duration as planned for the study. Both acute and chronic duration's isolation may be used. Neonatal isolation stress models were also developed wherein, maternal separation as early life stress experience was induced in neonates. These models help in understanding the neural and behavioral adverse effects on quality of life at a later stage of life [5]. Generally, the neonate was separated from its mother on the second day after birth and placed in a separate cage for duration of 30 to 60 minutes away from the room of other animals. Vocalization of other animals and pups from the vicinity is cut off by playing a background white noise as masking effect. Separation at least for an hour on daily basis and may be induced in multiples of days such as 4, 8 and 12 days. This early life isolation model is extensively adopted in the study of alcohol/drug addiction.

\subsection{Noise stress}

Noise is a destructive stimulus and present urban living people in many countries are constantly exposed to the motorized transport vehicles which emit noise at higher decibels than the healthy levels. Similarly, 
occupational exposure with the people working in such industries is also common and noise is a hazardous psychological stimulus. In animal models of noise stress studies, generally, loudspeakers are used along with white noise near the experimental animal set-up. Generally, these speakers are fixed around $30-40 \mathrm{~cm}$ from the experimental animal cage usually towards the roof of the cage. The noise limit is fixed at $100 \mathrm{~dB}$ to higher levels and animals may be exposed for short durations (acute) or repeatedly from 3 to $4 \mathrm{hrs}$ duration daily for a period of $5,10,15$ or 30 days depending on the design of the study [6]. Anxiety and depression on exposure to noise and the behavioral outcomes will be studied, in addition to the hormonal, physiological and molecular mechanisms that are involved in noise induced stress experiences $[7,8]$.

\subsection{Dark-light; circadian rhythm alteration stress}

Circadian rhythm is regulated by the biological clock in the hypothalamus. Day and night-shift workers are mainly exposed to these variations as their wakefulness and sleep cycles keep varying according to their shifts and also long-distance flight journey to different continents also have profound effects. Variations and alteration in the rhythm is known to produce psychological and physiological balance [9]. In this model, a reversal of circadian rhythm was artificially created by lighting the area of experimentation during natural dark or night. Electric bulbs are lit during the night from $7 \mathrm{pm}$ to $7 \mathrm{am}$ and day time with artificial darkness. Sometimes 1 to 3 hrs dark-light session also will be created. This reversal of the natural cycle acts as a stress stimulus but if the session should not be repeated for long durations as there will be adaptation. This is a good model of anxiety related stress studies as pineal gland and melatonin secretions have a profound role in the body in maintaining the homeostasis $[10,11]$.

\subsection{Predator exposure}

This model is an example to study the post-traumatic stress induced disorders (PTSD) in human being. Since there are limitations to study every symptom of PTSD in an animal model wherein nightmares or invasive thoughts cannot be examined. Predator exposure generally induces anxiety and hyper-arousal, the fearful manifestations in the animal can be comparable with PTSD subjects [12]. A predator such as a cat is suddenly brought in front of a rat for duration of 5 to 30 minutes and there are evidences of neurotransmitter alterations in specific brain areas such as amygdale, hippocampus and prefrontal cortex which are associated with PTSD [13].

\section{PHYSICAL OR SOMATIC STRESS MODELS}

\subsection{Immobilization stress}

It is also called as restraint stress. In experimental animals, immobilization or restraint is a most common type of stress induced to study the physiological, biochemical, hormonal and behavioral sequences. In this model, movement of the animal is totally restricted for a scheduled duration. Generally, animals were confined in cylindrical or semi cylindrical tubes or pipes with proper ventilation and sometimes gentle wrapping of fore and hind limbs to special made wooden boards by adhesive tapes. Head movement is also restricted and the duration of restrain vary from 1 - 3hrs on daily basis with an acute and chronic duration $[4,14]$. Immobilization results in both mental and physical stress with a high level of anxiety and lesser level of adaptation $[14,15]$.

\subsection{Hot and cold stress or temperature variation stress}

Thermostatic center in the hypothalamus keeps the body temperature within normalcy. Any change in the body temperature by means of altering the surrounding temperature either to cold or hot than the body temperature acts as a stress stimulus. Cold water or temporary exposure to freezer atmosphere or similarly to the hot or warm atmosphere leads to the activation of HPA axis $[16,17]$. Variations in temperature stress, either cold or hot are generally studied for both acute and chronic durations [17].

\subsection{Electric foot shock stress}

Experimental animals especially the rodent model is extremely sensitive to the electric shock stimuli wherein a mild level of shock treatment to their foot induce fear complex with a significant stress response. In this foot shock protocol, rat or mice will be placed in a cage with metal grid floor. Metal grid is connected to a shock generating instrument. Animals were given a mild shock of 0.5 to $2 \mathrm{~mA}$ for short duration of 1 to 2 seconds or may be also given intermittent shock [18].

\subsection{Chronic unpredictable stress in animal model}

This model is used to study the behavioral changes on exposure to a variety of stress stimuli with an unpredictable situation in the rodents. Generally, a stress stimulus is presented at random time on daily basis. In these unpredictable stress stimuli, stress adaptation is prevented. Wide variety of stressors such as diurnal variation, restraint, noise, foot shock, hot or cold etc, was given at random interval. This type of stress is well correlated to represent human being's day to day exposures and mostly act as a realistic comparison to extrapolate clinically $[19,20]$.

Overall, animal models that were developed over-time representing both somatic and psychological stress have yielded a grounding base in the study of stress research that has enriched to clinical models of anxiety, depression and post-traumatic stress disorders. They have also contributed at large towards the development of drugs in the treatment of varied psychological and psychiatric disorders that are common among the human beings in the modern century.

\section{CONCLUSION}

Experimental models of stress study and usage of a variety of models that represent psychological, somatic and a combination of both have yielded fruitful outcomes. Experimental animals have paved a way-forward in the stress research and they have been successfully adopted in understanding the basis of many mental disorders from anxiety, depression, mood disorders, schizophrenia to bipolar mental syndrome. The basis, neurochemistry and genetic basis of these conditions are heavily studied using these models and contributed a lot into the clinical science and therapeutics.

\section{REFERENCES}

[1] Heim, C., Nemeroff, C.B. 1999. The impact of early adverse experiences on brain systems involved in the pathophysiology of anxiety and affective disorders. Biological Psychiatry, 46 (2), 1509-1522.

[2] Mathew, S.J., Price, R.B., Chamey, D.S. 2008. Recent advances in the neurobiology of anxiety disorders: implications for novel therapeutics. American Journal of Medical Genetics Part A, 148C, 89-98.

[3] Magarinos, A.M., Deslandes, A., McEwen, B.S. 1999. Effects of antidepressants and benzodiazepine treatments on the dendritic structure of CA3 pyramidal neurons after chronic stress. European Journal of Pharmacology, 371 (2), 113-122.

[4] D'Souza, U.J., Nagaraja, H.S., D'Souza, V.M., D'Souza, A., Jeganathan, P.S. 2004. Effect of ethanol on stress induced cardiovascular alterations in rats. The Journal of Physiological Sciences, 17 (2), 80-85.

[5] Francis, D.D., Meaney, M.J. (1999). Maternal care and the development of stress responses. Current Opinion in Neurobiology, 9 (2), 128-134.

[6] Ravindran, R., Rathisamy, S.D., Samson, J., Senthilvelan, M. (2005). Noise-stress induced brain neurotransmitter changes and the effect of Ocimum sanctum (Linn) treatment in albino rats. Journal of Pharmacological Sciences, 98 (1), 354-360.

[7] File, S.E., Fernandes, C. (1994). Noise stress and the development of benzodiazepine dependence in the rat. Anxiety, 1 (2), 8-12.

[8] Caitlin, R.K., John, P.S. (2011). How and why environmental noise impacts animals: an integrative, mechanistic review. Ecology Letters, 2 (1), 1-10.

[9] Atcheson, J.B., Tyler, F.H. (1975). Circadian rhythm: man, and animals. In: Grrep RO, Astwood EB, editors. Handbook of physiology. Washington: American Physiological Society, 127-134.

[10] Rai, D., Bhatia, G., Sen, T., Palit, G. 2003. Comparative study of perturbations of peripheral markers in different stressors in rats. Canadian Journal of Physiology and Pharmacology, 81 (2), 1139-1146.

[11] Nicholson, S., Lin, J.H., Mahmoud, S., Campbell, E., Gillham, B., Jones, M. 1985. Diurnal variations in responsiveness of the hypothalamopituitary- adrenocortical axis of the rat. Neuroendocrinology, 40 (2), 217224.

[12] Pitman, R.K. 1997. Overview of biological themes in PTSD. Annals of the New York Academy of Sciences, 821 (2), 1-9. 
[13] Baisley, S.K., Cloninger, C.L., Bakshi, V.P. 2011. Fos expression following regimens of predator stress versus foot-shock that differentially affect pre-pulse inhibition in rats. Physiology and Behavior, 104 (2), 796803.

[14] Padovan, C.M., Guimaraes, F.S. 2000. Restraint induced hypoactivity in an elevated plus maze. Brazilian Journal of Medical and Biological Research, 33 (2), 79-83.

[15] Kasuga, S., Ushijima, M., Morihara, N., Itakura, Y., Nakata, Y. 1999. Effect of aged garlic extract (AGE) on hyperglycemia induced by immobilization stress in mice. Nihon Yakurigaku Zasshi, 114 (3), 191-197.

[16] O'Connor, P., Chipkin, R.E. 1984. Comparisons between warm and cold-water swim stress in mice. Life Sciences, 35 (2), 631-639.
[17] Jaggi, A.S., Bhatia, N., Kumar, N., Singh, N., Anand, P., Dhawan, R. 2011. A review on animal models for screening potential anti-stress agents. Neurological Sciences, 32 (2), 993-1005.

[18] Hermann, L., Ionescu, I.A., Henes, K., Golub, Y., Wang, N.S., Buell, D.R. 2012. Long lasting hippocampal synaptic protein loss in a mouse model of post-traumatic stress disorder. PLOS One 7: e42603.

[19] Santarelli, L., Saxe, M., Gross, C., Surget, A., Battaglia, F., Dulawa, S. 2003. Requirement of hippocampal neurogenesis for the behavioral effects of antidepressants. Science, 301 (2), 805-809.

[20] David, D.J., Samuels, B.A., Rainer, Q., Wang, J.W., Marsteller, D., Mendez, I. 2009. Neurogenesis -dependent and -independent effects of fluoxetine in an animal model of anxiety/depression. Neuron, 62 (2), 479493. 